\title{
A Wideband and a Wide - Beamwidth Acoustic Transducer Design for Underwater Acoustic Communications
}

\author{
I.Ceren EImaslı \\ Bilkent University, \\ Dept of Electrical and Electronics Engineering, Ankara, Turkey \\ elmasli@ee.bilkent.edu.tr \\ Hayrettin Köymen \\ Bilkent University, \\ Dept of Electrical and Electronics Engineering Ankara,Turkey \\ koymen@ee.bilkent.edu.tr
}

\begin{abstract}
This paper is concerned with the design of an efficient, wideband and a wide-beamwidth resonant acoustic transducer for high frequency use. The general transducer structure which has two back-to-back quarter wave thick 1 - 3 composite ceramic elements at resonance frequency is introduced. The transducer is employed for both transmit and receive modes. Design of transmitting and receiving transducers are discussed. Several transfer functions are derived and their effective bandwidths are calculated. It is shown that the phase angle difference between two acoustic ports in receive mode can be processed at the electrical ports to maintain better throughput. The paper includes future works to be done. It is concluded that the proposed structure can be used for applications of spread spectrum schemes in underwater communications.
\end{abstract}

\section{INTRODUCTION}

Employment of new mobile communication schemes based on spread spectrum techniques or their modified versions can provide a means for acoustic voice and data communications at short ranges [1] [2]. When employed at a high frequency range, these schemes provide advantages such as low power emission, and hence undetectability at a distance, as well as suitability for networking. On the other hand the applicability of such schemes has problems, where requirement for a wideband and wide - beamwidth transducer is one of them. Designing efficient, wideband and wide - beamwidth acoustic transducers has inherent barriers due to relatively large achievable physical dimensions of the transducer structures [3].

In this paper, we introduce a transducer model configured both as a transmitter and a receiver. The transducer is enhanced for exchanging voice and data in underwater media. The system is designed to operate in a half - duplex mode. The plots and results are generated by using Matlab ${ }^{\circledR}$.

The following section is concerned with the general structure of proposed transducer model. Transmitting and receiving transducer models are introduced. The models are discussed in details. Next, a design example is introduced. Several transfer functions are derived and examined. Finally, the features of transducer model are outlined, and future work is explained.

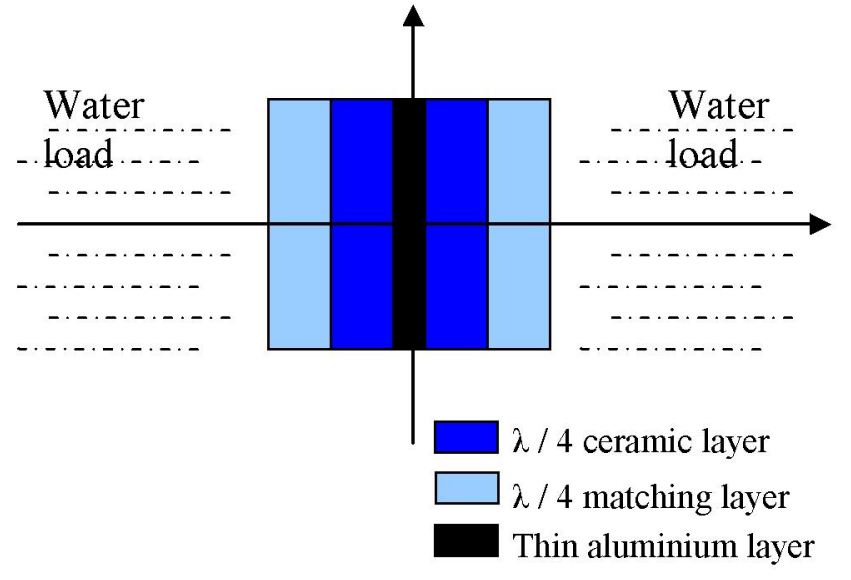

Fig. 1. General transducer structure made of $1-3$ composite ceramic, matching and aluminium layers.

\section{THE TRANSDUCER}

The general transducer structure is composed of matching, aluminium and $1-3$ composite ceramic layers as shown in Fig. 1. The two radiating faces (to each half space) of the transducer are displaced by the length of the structure. The displacement is about $2-2.5$ wavelengths in water at resonance frequency, with available materials. This configuration employs two electrical ports, $V_{1}$ and $V_{2}$ besides two acoustic ports, $F_{1}$ and $F_{2}$ as shown in Fig. 2.

We considered piezocomposite materials in the structure rather that ceramics because they offer increased sensitivity, broader bandwidth, improved impedance match to water and higher efficiency [4]. Composite piezoelectric materials can be prepared by combining a piezoelectric ceramic with a passive polymer phase [5] [6]. The piezoelectric materials have the ability of converting the electrical driving pulse into acoustical energy besides detecting the weak acoustical force and converting into electrical energy.

The transducer structure has two back-to-back quarter wave thick $1-3$ composite ceramic elements at its resonance frequency. Each element provides the "rigid", or high impedance backing to the other element, maintaining efficiency. This structure has advantages compared to a single half wavelength ceramic transducer which is matched to water 
on both faces, such that, this property is useful for deriving linear combinations of two separate signals which are received at the acoustic ports in receiving mode.

The ceramic layers are separated by a thin aluminium layer as shown in Fig. 1. In fact aluminium layer is not required from the performance point of view but it is included to provide a mounting support.

The general transducer structure, which is depicted in Fig. 1, is enhanced for both transmit and receive modes. In transmission mode, we connect electrical ports parallel to each other. The circuit of transmitting transducer is shown in Fig. 2. Aluminium and matching layers are presented by their transmission line equivalent models. We demonstrate $1-3$ composite ceramic layers with their Mason's equivalent circuit model [7]. Electrical ports $\mathrm{V}_{1}$ and $\mathrm{V}_{2}$ are connected to voltage source, $\mathrm{V}_{\mathrm{s}}$. Table I presents detailed data describing the circuit components shown in Fig. 2.

The equivalent circuit of transducer in receive mode is sketched in Fig. 3. We used additional feedback amplifiers at the electrical ports. They cancel the effect of positive capacitance due to "virtual ground".

The wide band characteristics of a transducer can be arranged by proper adjustment of length and impedance of the matching layer. In order to achieve a wideband transducer, we evaluated the admittance seen from the acoustic ports. In Fig. 4 and Fig. 5, admittance vs. normalized frequency graphs are sketched for different values of length and impedance of matching layer. It turned out that the $3-\mathrm{dB}$ effective bandwidth of a maximally flat admittance response (Fig. 4) doesn't achieve maximal bandwidth. We arranged the matching layer properties in order to perform maximal bandwidth by allowing an admittance variation down to $70 \%$ of its maximum value. The frequency spectrum shown in Fig. 5 is sketched with respect to frequency, $f_{0}$, which is the resonance frequency of a half - wavelength ceramic layer

We load the radiating faces of transducer with acoustic impedance of water during transmission. In a simple transmit and receive scenario, an impulse is generated at voltage source, $\mathrm{V}_{\mathrm{s}}$ and acoustic forces are produced at the radiating acoustic faces. Then the transmitter radiates acoustic forces, $F_{1}$ and $F_{2}$ which are equal in phase and magnitude, to two half spaces in the water channel. When force is detected at the acoustic ports of the receiver, it is converted into voltage and realized at the electrical ports. However, due to the length of the transducer, the front acoustical port of receiving transducer detects the transmitted force earlier than the rear one. There are a few points that we figure out in our propagation model. First, we consider the receiver is affected only from the acoustic signals that are generated from the front acoustic port of the transmitting transducer. Second, we assume the receiver is located at the far - field.

Since, receiving and transmitting transducers are not connected to each other; they can be positioned in an arbitrary direction and position. The signal strength and the phase that arrives at the receiver are also affected from respective alignments of receiving and transmitting transducers.

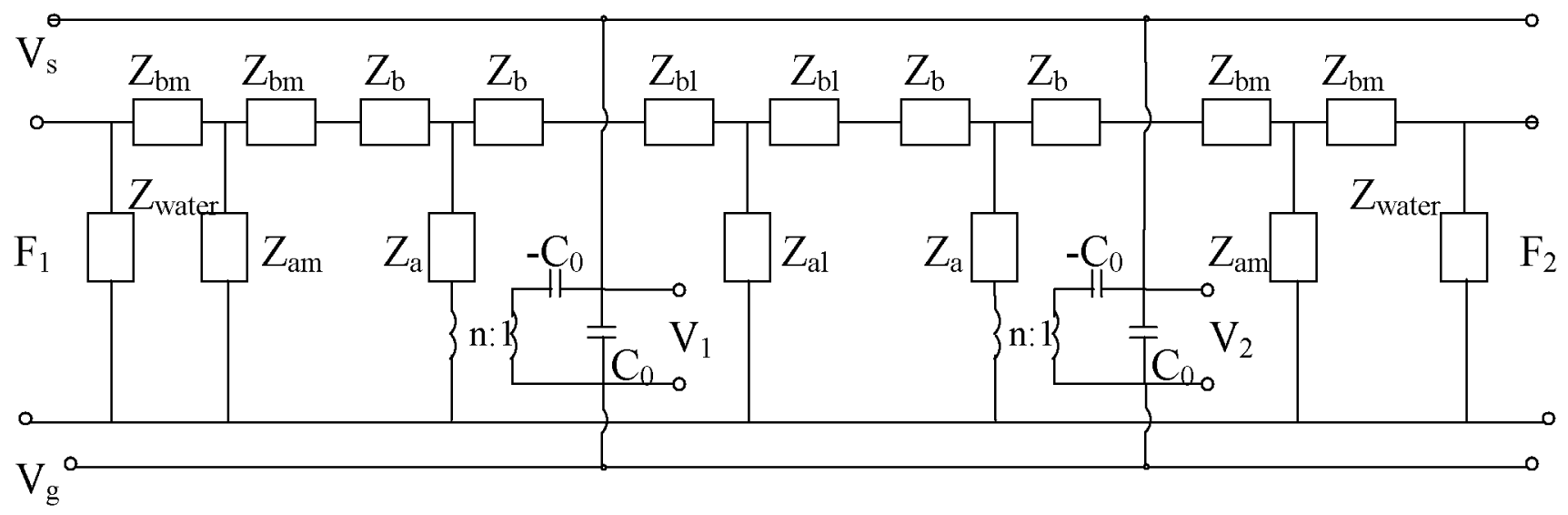

Fig. 2. The circuit diagram of transmitting transducer. Electrical ports are connected parallel to each other.

\begin{tabular}{|c|c|c|c|}
\hline & \multicolumn{3}{|c|}{$\begin{array}{c}\text { TABLE I } \\
\text { TRANSDUCER COMPONENTS }\end{array}$} \\
\hline & Matching layer & Piezocomposite layer & Aluminium layer \\
\hline Surface Area & $\mathrm{A}$ & $\mathrm{A}$ & $\mathrm{A}$ \\
\hline Length & $\mathrm{l}_{\mathrm{m}}$ & 1 & $\mathbf{l}_{\mathrm{al}}$ \\
\hline Wavelength & $\lambda_{\mathrm{m}}$ & $\lambda$ & $\lambda_{\mathrm{al}}$ \\
\hline Propagation constant & $\beta_{\mathrm{m}}$ & $\beta$ & $\beta_{\mathrm{al}}$ \\
\hline Density & $\mathrm{C}_{\mathrm{m}}$ & $\mathrm{C}$ & $\mathrm{C}_{\mathrm{al}}$ \\
\hline Acoustic Impedance & $Z_{\mathrm{m}}=\beta_{\mathrm{m}} \mathrm{C}_{\mathrm{m}} \mathrm{A}$ & $Z=\beta C A$ & $Z_{\mathrm{al}=} \boldsymbol{\beta}_{\mathrm{al}} \mathrm{C}_{\mathrm{al}} \mathrm{A}$ \\
\hline & $Z_{\mathrm{ma}}=-\mathrm{j}^{*} Z_{\mathrm{m}} * \operatorname{cosec}\left(\beta_{\mathrm{m}}{ }^{*} 1_{\mathrm{m}}\right)$ & $Z_{a}=-j * Z_{*} \operatorname{cosec}\left(\beta^{*} l\right)$ & $Z_{\mathrm{al}}=-\mathrm{j} * Z_{\mathrm{al}} * \operatorname{cosec}\left(\beta_{\mathrm{al}} * \mathrm{a}_{\mathrm{al}}\right)$ \\
\hline & $Z_{\mathrm{mb}}=\mathrm{j} * Z_{\mathrm{m}} * \tan \left(\beta_{\mathrm{m}} * 1_{\mathrm{m}} / 2\right)$ & $Z_{b}=j^{*} Z_{*} \tan \left(\beta^{*} 1 / 2\right)$ & $Z_{\mathrm{bl}}=\mathrm{j} * \mathrm{Z}_{\mathrm{a} 1} * \tan \left(\beta_{\mathrm{a} 1} * \mathrm{a}_{\mathrm{a} 1} / 2\right)$ \\
\hline
\end{tabular}




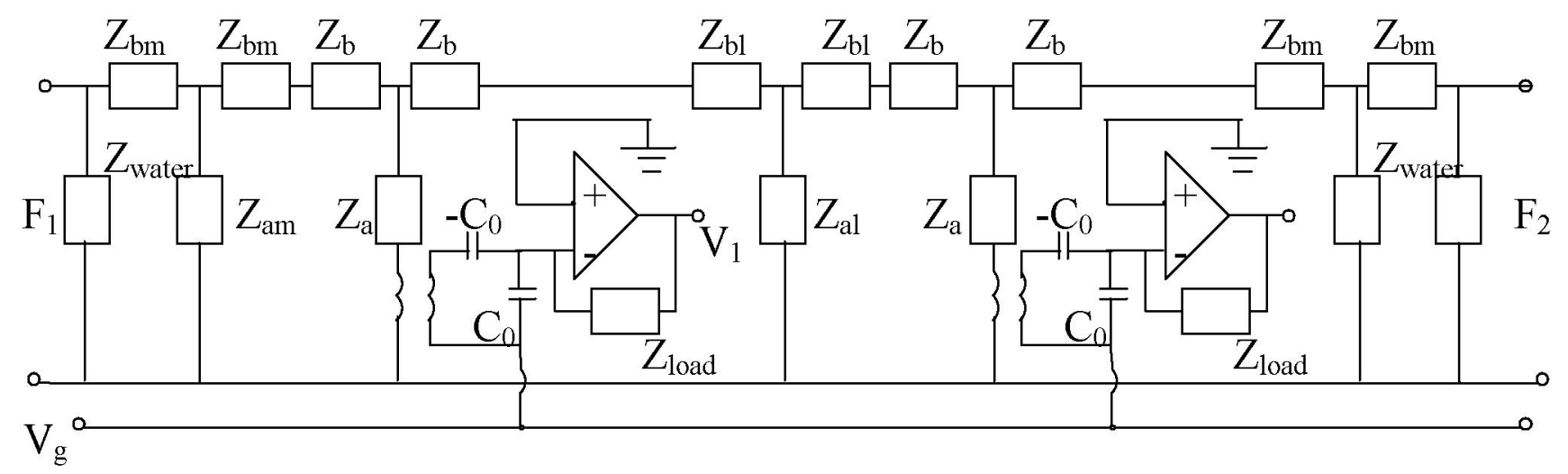

Fig. 3. The circuit diagram of receiving transducer. Feedback amplifiers are used.

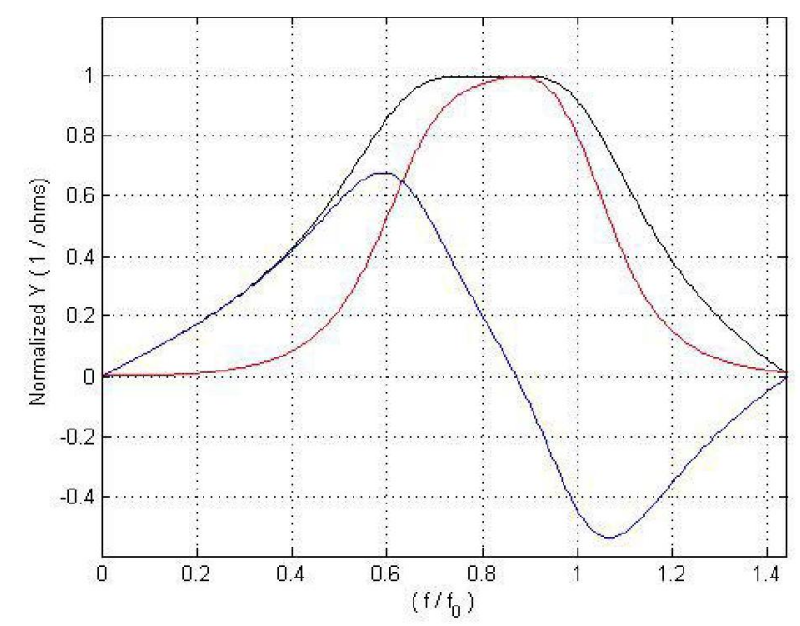

Fig. 4. Maximally flat normalized admittance graph.

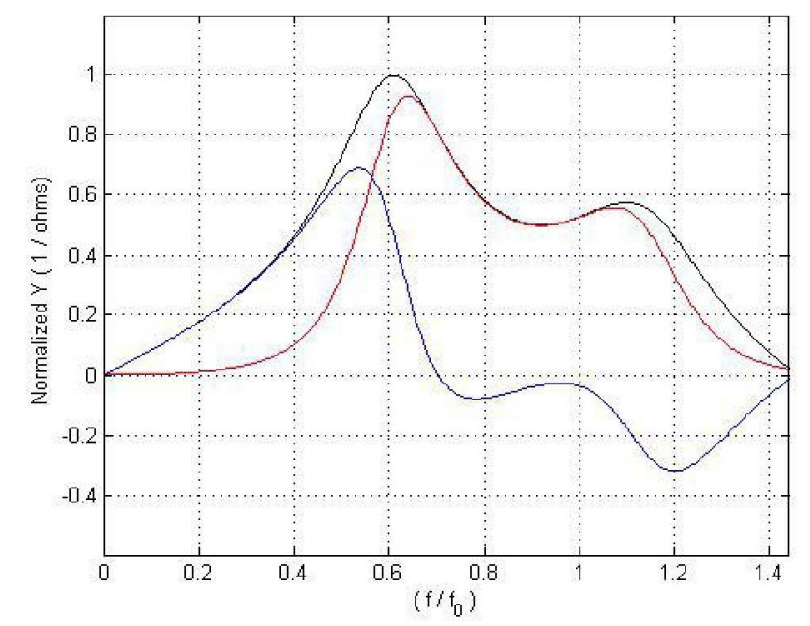

Fig. 5. Normalized maximal bandwidth admittance graph.

\section{DESIGN EXAMPLE}

The proposed transducer structure is employed both for receiving and transmitting purposes. The acoustic ports of receiving and transmitting transducers are placed parallel to each other. The distance between them is kept large ( $\sim 10$ meters). The resonance frequency, $f_{0}$, of a half wavelength ceramic layer, is $400 \mathrm{kHz}$.

The acoustic forces detected at the receiving transducer are called $F_{1}$ and $F_{2} . F_{1}$ is the force that is realized at the front acoustic port of the receiver. $F_{2}$ is the force that is realized at the rear port. Only, the effect of radiation from the front acoustic port is considered. Radiation from back plane of transmitter is not included in the analysis.

We use $30 \%$ PZT-5A and $70 \%$ stycast for the $1-3$ composite ceramic layers [8]. The layers are a quarter wavelength long, which are $2.875 \mathrm{~mm}$ at resonant frequency. Their impedance is 12.2 MRayl. The speed of sound in the material is $4600 \mathrm{~m} / \mathrm{sec}$.

The matching layers are kept around a quarter wavelength long at the resonance frequency of transducer. The characteristic impedance of matching layer is found to be 3.3 MRayl.

The aluminium layer that separates the $1-3$ composite ceramic layers is $1 \mathrm{~mm}$ long. The layer has an impedance of 16.2 MRayl. The speed of sound in aluminium layer is $6000 \mathrm{~m} / \mathrm{sec}$ and its density is $2700 \mathrm{~kg} / \mathrm{m}^{3}$.

The transducer is about $8 \mathrm{~mm}$ long at its center frequency.

We modeled each transducer square shaped, because they are easy to fabricate. The surface area of each element is $9 * 10^{-6} \mathrm{~m}^{2}$.

We drive the electrical ports of transmitter shown in Fig. 2 with unit impulse. One - way and two way transfer functions are examined. One - way transfer function is defined as the impulse response of the transmitting transducer. Two - way transfer function refers to the response of the receiving transducer to the impulse which is generated at the voltage source of transmitting transducer.

Fig. 6 shows the one - way transfer function, $F_{1} / V_{s}$, versus normalized frequency, ' $\mathrm{f} / \mathrm{f}_{0}$ '. The transfer function employs two peaks which are not symmetric. We preferred to use a non - symmetric impulse function, because the symmetry decreases the effective bandwidth. The function shown in Fig. 6 has an effective bandwidth of $85 \%$. It is analyzed that the center frequency of the transducer is less than the 
resonance frequency of a half wavelength ceramic. The clamped capacitance, $\mathrm{C}_{0}$, decreases the center frequency.

The acoustic waves radiated from acoustical ports of transmitter reach acoustic ports of receiving transducer and create electrical signals at the electrical ports. The two way transfer function $V_{1} / V_{s}$ when $F_{2}=0$, is shown in Fig. 7. Even though the real part of the function employs a null value, the effective bandwidth of absolute transfer function is $69 \%$. Fig. 8 shows the two way transfer function $V_{1} / V_{s}$ when $F_{1}=0$. Its effective bandwidth is $72 \%$.

The two way transfer function $V_{2} / V_{s}$ when $F_{2}=0$, is shown in Fig. 9. The real part of the functions passes zero three times through the sketched frequency range. Its effective bandwidth is $72 \%$. Fig. 10 shows the two way transfer function $V_{2} / V_{s}$ when $F_{1}=0$. Its effective bandwidth is $67 \%$. The real part of the functions passes zero three times through the sketched frequency range.

Fig. 11 and Fig. 12 show the voltage values that are determined at the electrical ports $V_{1}$ and $V_{2}$ when an impulse is generated at the transmitter. Both two transfer functions have the same effective bandwidth, $71 \%$. The functions are equal in magnitude. However, there is phase difference in between them. This is due to the distance in between two radiating faces. Acoustic signals reach the rear receiving face $5.46 \mu \mathrm{sec}$ after they reach the front receiving face. This value is consistent with the length of the transducer.

\section{DISCUSSION}

The configuration mentioned in the previous part reveals the transducer performance when radiating and receiving acoustic ports lie on the same plane. It is found out the functions $V_{1} / V_{s}$ and $V_{2} / V_{s}$ shown in Fig. 11 and Fig. 12 differ by a constant phase value. We decided to add the phase difference to the $V_{1} / V_{s}$ function. When the function $V_{2} / V_{s}$ is added to the delayed function $V_{1} / V_{s}$, the resultant response becomes as shown in Fig. 13. It is seen that this approach increases the magnitude of received voltage in a constructive manner. When we changed the distance between transmitter and receiver keeping the transmit and receive faces parallel to each other, we analyzed that the absolute magnitude of the function is not affected. However, the phase is changed with respect to the distance.

This work has presented that the proposed transducer structure provides a good potential for application of spread spectrum schemes in underwater communications.

Radiation from back plane of the transmitter is not included to the previous analysis. For future work, the effect of rear force will be included.

We will analyze the effect of different receiver orientations and positions in water media.

Three orthogonal transducers will be used in the structure instead of one for wide beam coverage and space diversity. Its propagation characteristics will also be investigated.

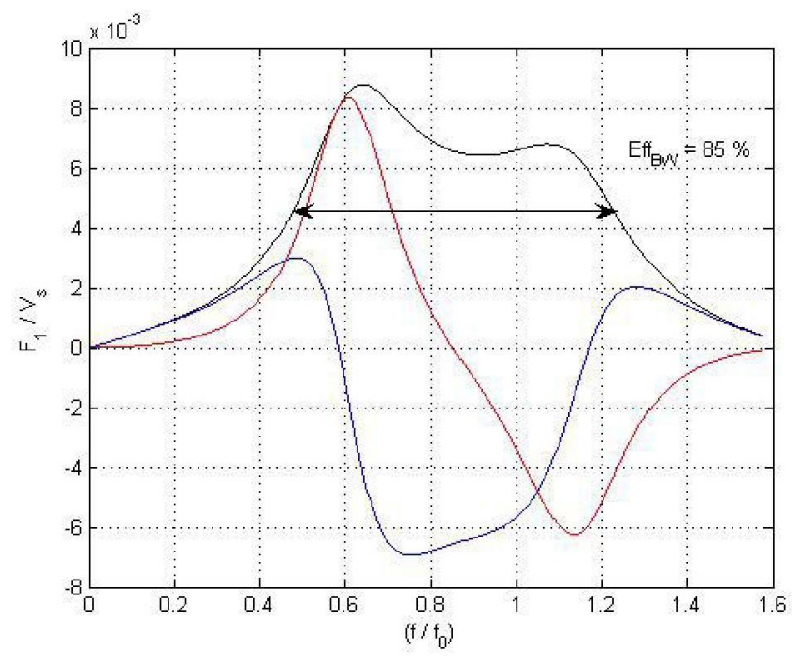

Fig. 6. One way transfer function of $F_{1} / V_{s}$. Red line represents real part, blue line represents the imaginary part. Black line is the absolute value of the transfer function.

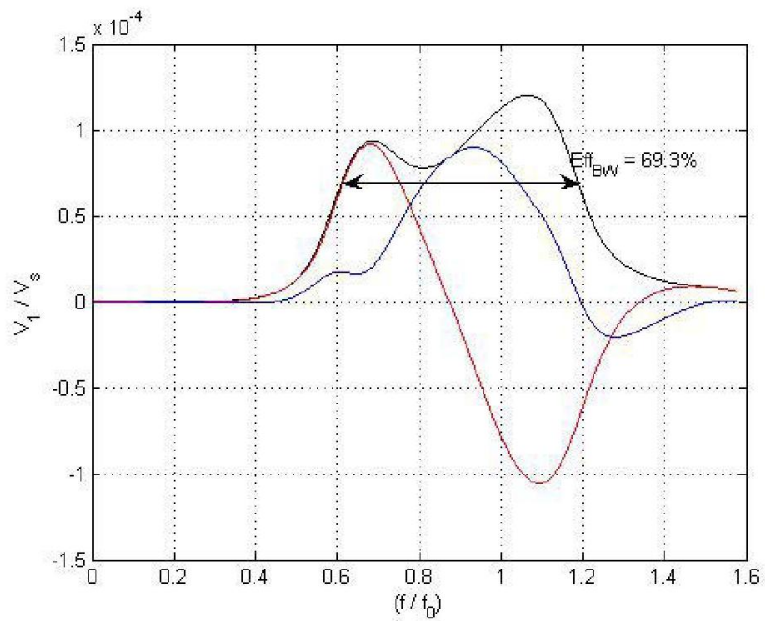

Fig. 7. Two way transfer function $V_{1} / V_{s}$ where $F_{2}=0$.

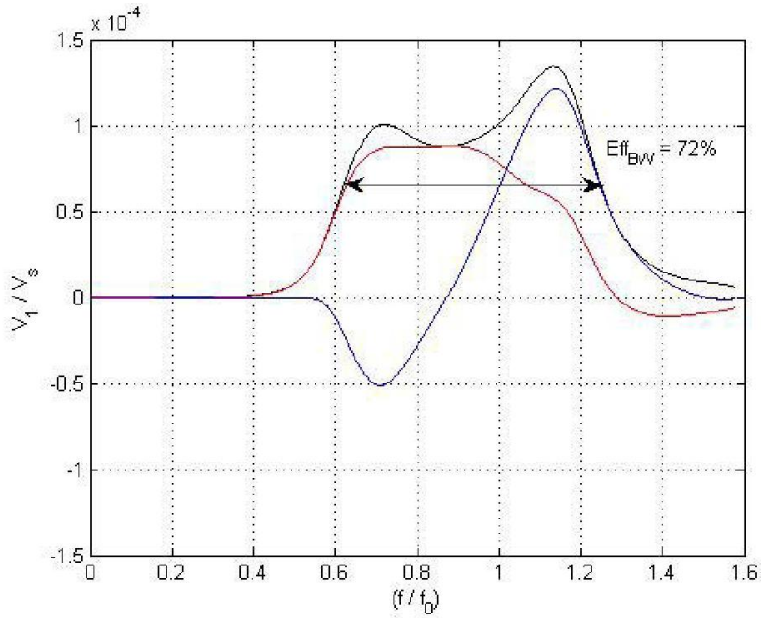

Fig. 8. Two way transfer function $V_{1} / V_{s}$ where $F_{1}=0$. 


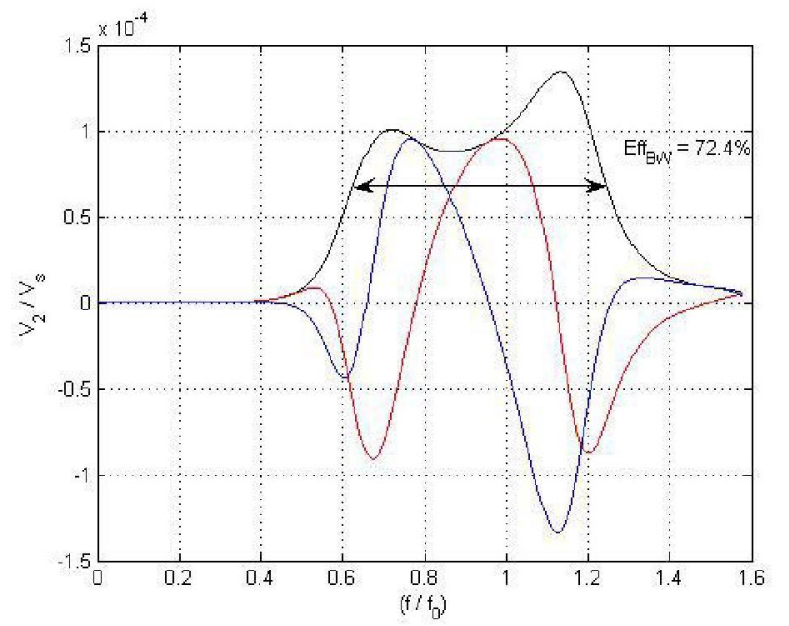

Fig. 9. Two way transfer function $V_{2} / V_{s}$ where $F_{2}=0$.

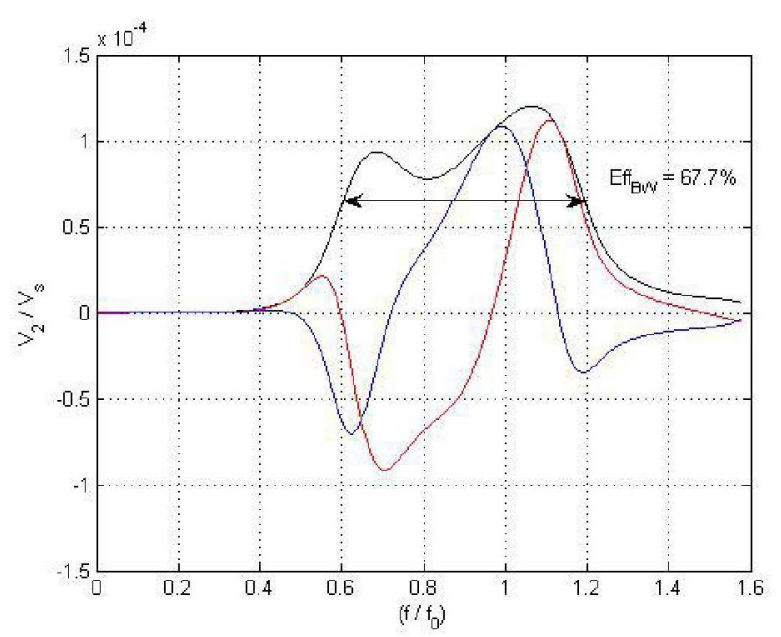

Fig. 10. Two way transfer function $V_{2} / V_{s}$ where $F_{1}=0$.

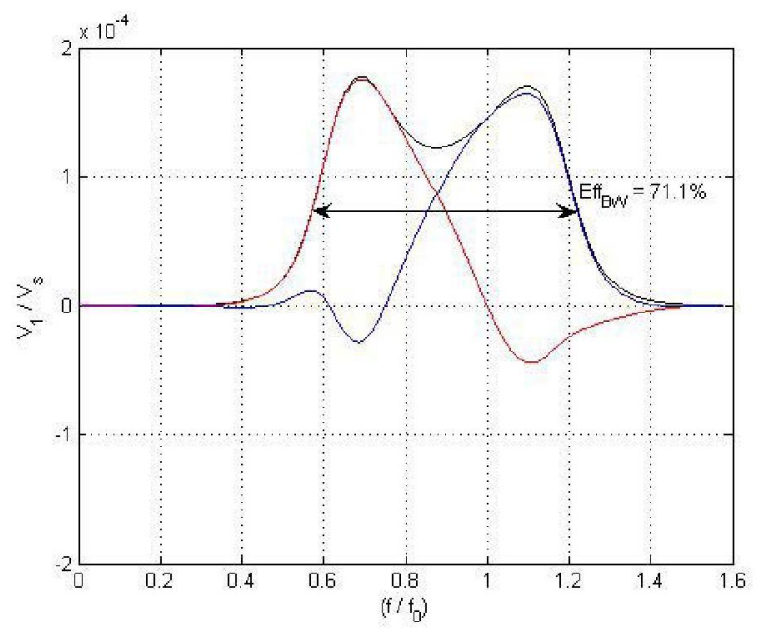

Fig. 11. Overall transfer function $V_{1} / V_{s}$.

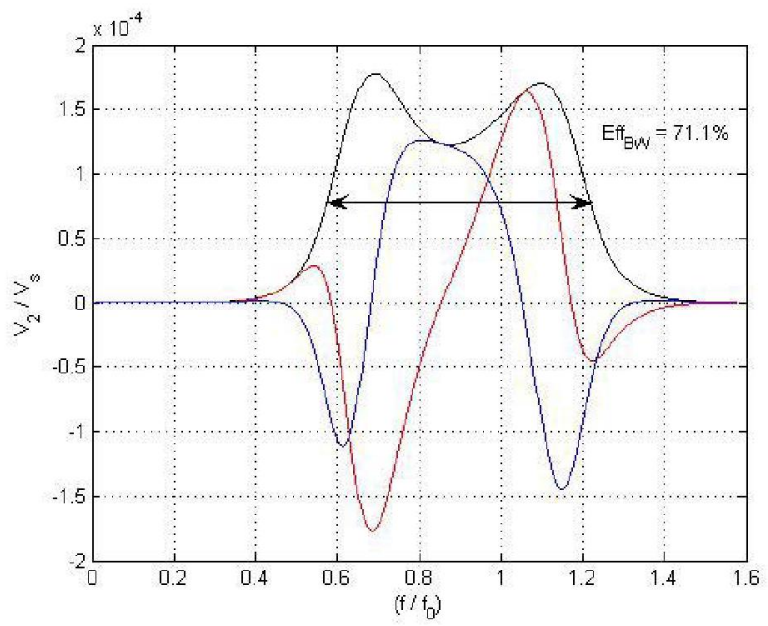

Fig. 12. Overall transfer function $V_{2} / V_{s}$.

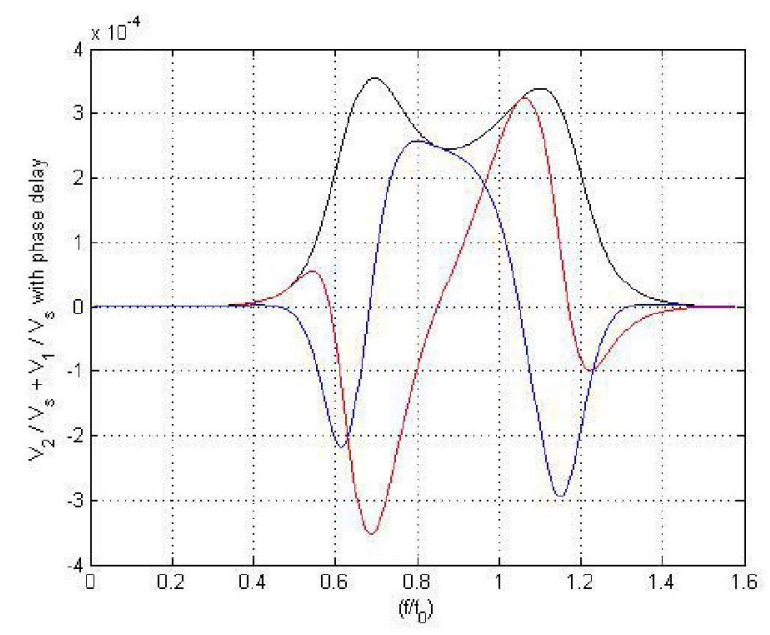

Fig. 13. Addition of $V_{2} / V_{s}$ and phase delayed $V_{1} / V_{s}$.

\section{REFERENCES}

[1] Ethem M. Sozer, Milica Stojanovic, John G. Proakis, "Underwater Acoustic Networks", IEEE J. Oceanic Eng., vol 25, pp.72-83, Jan. 2000.

[2] B. Woodward, H. Sari, "Digital underwater voice communications", IEEE J.'Oceanic Eng., vol 21, pp.181 192, Apr. 1996.

[3] Rodney F. W. Coates, "Design of transducers and arrays for underwater data transmission", IEEE J. Oceanic Eng., vol 16, pp.123 - 135, Jan. 1991.

[4] http://Www.matsysinc.com/downloads/TransducerApplicat ions.pdf

[5] W. A. Smith, "Modeling $1-3$ composite piezoelectrics: Thickness mode oscillations", IEEE Trans. Ultrason. Ferroelec. Freq. Contr., vol. 38, pp. 40 - 46, 1991.

[6] W. A. Smith, "The role of piezocomposites in ultrasonic transducers", 1989 IEEE Ultrason. Symp., pp. 755 - 766, 1989.

[7] Gordon S. Kino, "Acoustic waves: Devices, imaging and analog signal processing", $1^{\text {st }}$ Ed., p. $27-35$, New Jersey: Prentice Hall, 1987.

[8] W. A. Smith, "Modeling $1-3$ composite piezoelectrics: Hydrostatic Response", IEEE Trans. Ultrason. Ferroelec. Freq. Contr., vol. 40, pp. $41-49,1993$. 